\title{
The Hydrogen Laminar Jet
}

\author{
M. Sánchez-Sanz ${ }^{\mathrm{a}, *}$, M. Rosales ${ }^{\mathrm{b}, \mathrm{c}}$, A. L. Sánchez ${ }^{\mathrm{b}}$ \\ ${ }^{a}$ Departamento de Motopropulsión y Termofluidomecánica, ETSI Aeronáuticos, Universidad Politécnica de Madrid, \\ Madrid, Spain \\ ${ }^{b}$ Dept. Ingeniería Térmica y de Fluidos, Universidad Carlos III de Madrid, 28911, Leganés, Spain \\ ${ }^{c}$ Instituto de Innovación en Minería y Metalurgia, Avenida del Valle 738, Santiago, Chile
}

\begin{abstract}
Numerical and asymptotic methods are used to investigate the structure of the hydrogen jet discharging into a quiescent air atmosphere. The analysis accounts in particular for the variation of the density and transport properties with composition. The Reynolds number of the flow $R_{j}$, based on the initial jet radius $a$, the density $\rho_{j}$ and viscosity $\mu_{j}$ of the jet and the characteristic jet velocity $u_{j}$, is assumed to take moderately large values, so that the jet remains slender and stable, and can be correspondingly described by numerical integration of the continuity, momentum and species conservation equations written in the boundary-layer approximation. The solution for the velocity and composition in the jet-development region of planar and round jets, corresponding to streamwise distances of order $R_{j} a$, is computed numerically, along with the solutions that emerge both in the near field and in the far field. The small value of the hydrogen-to-air molecular weight ratio is used to simplify the solution by considering the asymptotic limit of vanishing jet density. The development provides at leading order explicit analytical expressions for the far-field velocity and hydrogen mass fraction that describe accurately the hydrogen jet near the axis. The information provided can be useful in particular to characterize hydrogen discharge processes from holes and cracks.
\end{abstract}

\section{Introduction}

The increased interest in hydrogen utilization promotes attention on safety concerns related to hydrogen storage. The accidental appearance of small holes or cracks in pressurized containers may lead to hydrogen jet-like discharge, leading to the formation of flammable mixtures of hydrogen and air in the surrounding atmosphere. To asses this accidental scenario, there is interest in characterizing the resulting discharging jets, accounting for the large density differences associated with the small weight of the hydrogen molecule, which cause the associated solution to be markedly different from that of a constant-density jet.

Different flow configurations relevant to hydrogen leakage have been considered in recent works [1, 2]. For instance, the far region of a concentration layer adjacent to a ceiling wall was addressed in [1], with constant density and viscosity assumed for the gas mixture. The similarity

\footnotetext{
${ }^{*}$ Corresponding author. Tel: +34 913363940 , Fax: +34 913363295

Email addresses: mario.sanchez@upm.es (M. Sánchez-Sanz ), mrosa001@im2.codelco.cl (M. Rosales ), asanchez@uc3m.es (A. L. Sánchez) 
description of a hydrogen buoyant jet was investigated in [2] in the Boussinesq approximation. In defining the relevant similarity variables, this latter investigation employes semi-analitical expressions derived in previous experimental works. Just as the previous studies, the present analysis aims at providing increased understanding of a relevant hydrogen flow configuration, leading to analytical descriptions that can be used in characterizing hydrogen discharge processes. In particular, numerical and asymptotic methods are used herein to investigate nonbuoyant hydrogen jets discharging into a quiescent air atmosphere.

When the Reynolds number $R_{j}=\rho_{j} u_{j} a / \mu_{j}$, based on the density and viscosity of the jet, $\rho_{j}$ and $\mu_{j}$, the characteristic initial jet velocity $u_{j}$, and the transverse dimension $a$, is sufficiently larger than unity, the resulting flow becomes slender, with a development length of order $\operatorname{Re}_{j} a \gg$ $a$, so that the associated solution can be described with small relative errors of order $R_{j}^{-2}$ by numerical integration of the boundary-layer equations, as done for instance in [3] in the constantdensity case. The corresponding solution applies to steady laminar flows, which therefore limits the applicability of the results to configurations with moderately large values of the Reynolds number below the critical value at which the flow becomes unstable. Even though the theoretical stability studies $[4,5]$ indicate that the onset of instability occurs for constant density flows at a critical Reynolds numbers given by $R e_{j} \simeq 30$ and $R e_{j} \simeq 40$ for planar and round jets, respectively, the experimental evidence seems to suggest that the critical values are considerably larger. For instance, in the early work of Andrade and Tsien [6] the laminar steady solution was found to exist for Reynolds numbers as large as $R e_{j} \sim 300$ and laminar jets have been obtained in more recent experiments for values of $R e_{j}$ exceeding $R e_{j}=600$ [7] and even $R e_{j}=1000$ [8]. It is generally agreed that the development of the instability is slow, so that for values of the Reynolds number on the order of a few hundred unsteadiness is only noticeable far downstream, at very large distances on the order of a few hundred nozzle diameters $[9,10]$, whereas the laminar steady solution remains valid at smaller distances from the jet exit. In the planar case, the resulting solution is more unstable than that of the round jet, as was experimentally confirmed in the early work of Andrade [11], who found the critical Reynolds number to be $R e_{j} \simeq 40$. Clearly, although the stability boundaries are expected to be modificed in the presence of density differences, the laminar steady results presented below can be expected to be applicable for the description of configurations with moderately large values of $R e_{j}$. The ideas developed below could also find application in simplifying the description of very light turbulent gas jets, an issue that should be investigated in the future.

The jet is initially separated from the outer stagnant flow by a mixing layer that grows from the injector rim, so that at distances of order $R_{j} a$ the action of molecular diffusion across the jet is seen to modify significantly the velocity and composition at the axis. As shown by Schlichting for the round jet [12] and by Bickley for the planar jet [13], the flow downstream from this development region approaches a self-similar solution corresponding to the flow induced by a point source of momentum. When the jet-gas properties are different from those of the surrounding atmosphere, the mixing process in the jet development region is modified through the dependence of the density and transport properties on the composition and temperature. The modifications can be very significant in cases where the differences between the jet gas and the ambient gas are large, as occurs for instance in the presence of large temperature differences $[14,15]$ or large molecular weight differences, the latter being the case of a hydrogen jet discharging into air.

The early investigations on the mixing of a gas jet with an atmosphere of a different gas are summarized in the book of Pai [16]. The problem of mixing of two streams of different gases was addressed in the early work of Chou [17], who employed for the first time the equation for the diffusion of the two gases to analyze mixing in the near-field mixing layer. Numerical results 
obtained for the mixing of gases with different density in laminar and turbulent flow conditions are reported in [16]. For a light jet, it was found that the rate of radial diffusion of mass is faster than that of momentum. Correspondingly, in light jets the concentration decays faster than the velocity along the jet axis. In the far-field, where the density differences are small, the asymptotic solutions of Schlichting [12] and Bickley [13] apply in the first approximation for the velocity field. The accompanying composition fields were determined by Crane and Pack [18] and their results where subsequently confirmed experimentally by Maydew and Reed [20].

Unlike previous works, full account of the density and viscosity variation with the hydrogen mass fraction is considered herein in integrating the boundary-layer equations. The analysis reveals that in the development region the solution for the hydrogen jet near the axis is independent of the density and viscosity of the air. The solution can be described by using the hydrogen-to-air molecular-weight ratio as an asymptotically small parameter, an analysis that provides in particular explicit analytical expressions for the decay of the velocity and hydrogen concentration in the intermediate zone downstream from the development region where the jet is still much lighter than the ambient. The comparisons with the numerical solutions indicate that these analytical solutions give an accurate representation for the hydrogen jet, which can be in particular useful in characterizing hydrogen discharge processes from small holes and cracks.

The relative effect of buoyancy on the structure of a hydrogen jet is measured by the relevant jet Froude number $F_{j}=u_{j}^{2} /\left(g a R_{j}\right)$, obtained as the ratio of the characteristic values of the acceleration and the gravity force in the jet development region, whose characteristic length is $R_{j} a$ for the large Reynolds numbers considered here. The buoyancy-free description given below therefore applies only to jets with relatively large values of the Froude number $F_{j} \gg 1$ such that gravity can be neglected in the first approximation when describing the velocity and composition fields at donwstream distances of order $R_{j} a$. Since the momentum-controlled velocity decreases with axial distance from the jet exit $x^{\prime}$, the effect of buoyancy can be expected to become nonnegligible sufficiently far downstream. In particular, according to the scalings identified below in (18), the gravity force becomes comparable to the flow acceleration at distances $x^{\prime} /\left(R_{j} a\right) \sim F_{j}^{1 /(2+i)}$. The corresponding plume flow, which should be analyzed in future work, would include a downstream region with small density variations where the flow that emerges will be identical to that of a jet with small temperature increments from the ambient temperature and small Prandtl numbers, a case analyzed earlier by Crane [21].

In view of the discussion given below, it is clear that the validity of the buoyancy-free slender solution presented below is restricted to configurations where the two conditions $R_{j} \gg 1$ and $F_{j} \gg 1$ are simultaneously satisfied. Note that, for a given jet radius, these two conditions can be written in the form $u_{j} \gg \mu_{j} /\left(\rho_{j} a\right)$ and $u_{j} \gg g a^{2} \rho_{j} / \mu_{j}$ thereby providing lower limits for the jet velocity that can be readily used to check the applicability of the proposed description. For instance, with $\mu_{j} / \rho_{j} \simeq 1 \mathrm{~cm}^{2} / \mathrm{s}$ for hydrogen at normal atmospheric conditions, it can be easily seen that for a jet of radius $a=0.1 \mathrm{~cm}$, both criteria are satisfied provided $u_{j}$ be sufficiently larger than $10 \mathrm{~cm} / \mathrm{s}$.

\section{Formulation}

As previously mentioned, for moderately large values of the Reynolds number, the jet remains slender and stable, and is therefore amenable to a boundary-layer description in which axial diffusion and transverse pressure gradients can be neglected with small relative errors of order $R_{j}^{-2}$. The problem can be formulated in nondimensional form using the scales corresponding to 
the development region. In particular, the jet momentum flux $J$ is used to define a characteristic jet velocity

$$
u_{j}=\left(\frac{J(i+1)}{2 \pi^{i} \rho_{j} a^{i+1}}\right)^{1 / 2}
$$

for round $(i=1)$ and planar $(i=0)$ jets. Note that this characteristic value is defined such that it becomes exactly equal to the inlet jet velocity in cases where the velocity profile is uniform. The value $u_{j}$ is used to scale the axial velocity $u=u^{\prime} / u_{j}$, while the transverse velocity $v^{\prime}$ is scaled with $\mu_{j} /\left(a \rho_{j}\right)$ to give the dimensionless variable $v$, with the primes denoting dimensional variables. The jet values are used to define the dimensionless density $\rho=\rho^{\prime} / \rho_{j}$ and viscosity $\mu=\mu^{\prime} / \mu_{j}$. Furthermore, the transverse coordinate $r^{\prime}$ is scaled with the characteristic transverse dimension $a$ (the initial radius for the round jet and the initial half-width for the planar jet) and the axial coordinate $x^{\prime}$ is scaled with the characteristic length of jet development $R_{j} a$, yielding the dimensionless coordinates $r=r^{\prime} / a$ and $x=x^{\prime} /\left(R_{j} a\right)$, respectively.

Attention is restricted to configurations in which the jet temperature equals that of the ambient. Furthermore, the jet velocity is assumed to be much smaller than the sound velocity, so that the effect of viscous dissipation on the energy balance can be neglected in the first apprximation. Under those low-Mach-number conditions, the temperature remains uniform in the flow field and the problem reduces to that of integrating

$$
\begin{aligned}
\frac{\partial \rho r^{i} u}{\partial x}+\frac{\partial \rho r^{i} v}{\partial r} & =0 \\
\rho u \frac{\partial u}{\partial x}+\rho v \frac{\partial u}{\partial r} & =\frac{1}{r^{i}} \frac{\partial}{\partial r}\left(r^{i} \mu \frac{\partial u}{\partial r}\right) \\
\rho u \frac{\partial Y}{\partial x}+\rho v \frac{\partial Y}{\partial r} & =\frac{1}{r^{i}} \frac{\partial}{\partial r}\left(r^{i} \frac{\rho}{S} \frac{\partial Y}{\partial r}\right)
\end{aligned}
$$

with boundary conditions for $x>0$

$$
\begin{aligned}
r=0 & : \quad \frac{\partial u}{\partial r}=v=\frac{\partial Y}{\partial r}=0 \\
r \rightarrow \infty & : \quad u=Y=0
\end{aligned}
$$

and initial conditions at $x=0$

$$
\begin{aligned}
0 \leq r \leq 1: & : \quad u-u_{i}(r)=Y-1=0 \\
r>1: & : \quad u=Y=0
\end{aligned}
$$

The initial velocity distribution $u_{i}(r)$ depends on the shape of the velocity profile at the jet exit. Cases of interest are the uniform profile $u_{i}(r)=1$ and the fully developed profile $u_{i}(r)=B\left(1-r^{2}\right)$, with $B=(\sqrt{15 / 8}, \sqrt{3})$ for $i=(0,1)$. Fickian diffusion of hydrogen is assumed in writing (4), with $S=\mu_{j} /\left(\rho_{j} D\right)=1.39$ being the relevant Schmidt number and $D$ representing the $\mathrm{H}_{2}$-air binary diffusion coefficient, constant for the isothermal and isobaric conditions considered here [22]. In the formulation, $Y$ denotes the hydrogen mass fraction. Correspondingly, the isothermal equation of state becomes

$$
\rho=\frac{1}{Y+\varepsilon(1-Y)}
$$

where $\varepsilon \simeq 0.07 \ll 1$ is the hydrogen-to-air molecular weight ratio. 


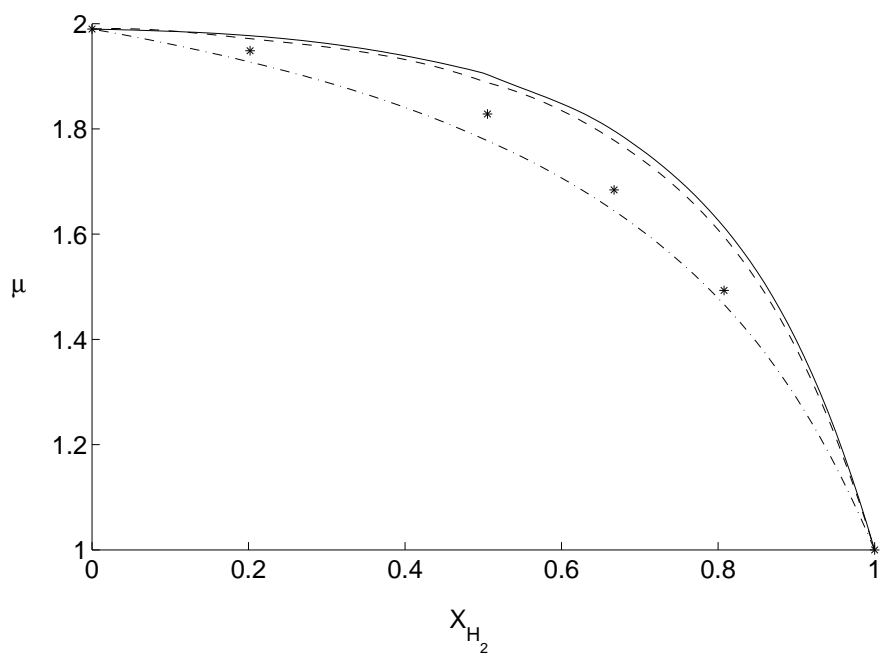

Figure 1: Comparison of measured and calculated viscosity for different $\mathrm{H}_{2}$ molar fractions $X_{\mathrm{H}_{2}}$. The curves represent computations with approximate formulae (dashed: Hirshfelder et al. [22]; dot-dashed: Rosner [23]; solid: Wilke [24]), while the symbols are the experimental measurements found in [22].

To close the problem, the variation of the viscosity with the mixture composition needs to be specified. The semi-empiric expression developed by Rosner [23]

$$
\mu=\frac{Y+(1-Y) \varepsilon^{1 / 2} \mu_{a}}{Y+(1-Y) \varepsilon^{1 / 2}}
$$

is used in the computations below, where $\mu_{a}=\mu_{a}^{\prime} / \mu_{j}=1.944$ is the air-to-hydrogen viscosity ratio. The accuracy of this expression is tested in Fig. 1 by comparison with the experimental data obtained for $\mathrm{H}_{2}$-air mixtures [22], yielding reasonable agreement over the whole range of hydrogen mole fraction $X_{\mathrm{H}_{2}}=Y /[Y+\varepsilon(1-Y)]$. The figure also exhibits the viscosity variation obtained with the semi-theoretical model of Wilke [24] along with the fully theoretical expression derived by Hirschfelder et al. [22]. Since these two alternative descriptions, which involve algebraic expressions that are more complicated than (8), do not provide improved accuracy, the simpler expression (8) is preferred for our jet computations.

Note that in the limit of very light jets $\varepsilon \ll 1$, the expression (8) simplifies to $\mu=1+$ $\varepsilon^{1 / 2}(1-Y)\left(\mu_{a}-1\right) / Y+O(\varepsilon)$, indicating that in regions where $Y \gg \varepsilon^{1 / 2}$ the viscosity is, in the first approximation, that of the light gas. Also of interest is that, since the viscosity $\mu$ remains of order unity regardless of the composition, transverse molecular diffusion of momentum in (3) is of comparable magnitude all across the jet. The diffusion velocity of the light species is however linearly proportional to the mixture density, and therefore increases as the hydrogen mass fraction decays away from the axis. The effective diffusion coefficient $\rho / S$, of order unity where $Y \sim O(1)$, becomes of order $\varepsilon^{-1} \gg 1$ where $Y \sim \varepsilon$, thereby promoting significantly the radial diffusion of the light species into the ambient air.

Before proceeding with the analysis, it is of interest to integrate radially the momentum and species conservation equations, once written in conservative form with use made of (2), to yield 
the integral constraints

$$
\int_{0}^{\infty}(1+i) \rho r^{i} u^{2} \mathrm{~d} r=1 \text { and } \int_{0}^{\infty}(1+i) \rho r^{i} u Y \mathrm{~d} r=q,
$$

to be satisfied by the solution of (2)-(4). The constant

$$
q=(1+i) \int_{0}^{1} r^{i} u_{i} \mathrm{~d} r
$$

represents the initial volume flux and takes the value $q=1$ with uniform inlet velocity profile and $q=(\sqrt{5 / 6}, \sqrt{3} / 2)$ for $i=(0,1)$ with parabolic inlet velocity profile.

\section{The hydrogen jet}

The solution for the hydrogen jet can be described numerically by integrating the boundary layer equations (2)-(4) with the boundary conditions given in (5) and the initial conditions given in (6). To facilitate the convergence of the solution near the jet exit, the initial conditions were replaced with the profiles of velocity and hydrogen mass fraction that appear for $x \ll 1$, where an annular mixing layer forms at $r=1$ between the jet and the outer stagnant fluid, as described separately below.

\subsection{The hydrogen-air mixing layer}

The solution in the initial mixing layer depends in general on the value of the inlet-velocity gradient $A=-\mathrm{d} u_{i} / \mathrm{d} r$ at $r=1$. This gradient equals $A=2 B$ for the parabolic profile and becomes larger for nonparabolic inlet velocity profiles with decreasing values of the boundarylayer thickness. The initial, Goldstein region, of the mixing layer can be described by introducing a similarity variable $\eta=(r-1) /(x / A)^{1 / 3}$ together with a stream function $\psi=A^{1 / 3} x^{2 / 3} F(\eta)$, defined such that $\rho u=A^{2 / 3} x^{1 / 3} F_{\eta}$ and $\rho v=A^{1 / 3} x^{-1 / 3}\left(\eta F_{\eta} / 3-2 F / 3\right)$, where the subscript $\eta$ indicates differentiation with respect to this variable. The problem reduces to that of integrating

$$
\begin{aligned}
{\left[\mu\left(\frac{F_{\eta}}{\rho}\right)_{\eta}\right]_{\eta}+\frac{2}{3} F\left(\frac{F_{\eta}}{\rho}\right)_{\eta}-\frac{1}{3} \frac{F_{\eta}^{2}}{\rho} } & =0 \\
\left(\rho Y_{\eta}\right)_{\eta}+\frac{2}{3} S F Y_{\eta} & =0
\end{aligned}
$$

subject to the boundary conditions $F+\eta^{2} / 2 \rightarrow 0$ and $Y \rightarrow 1$ as $\eta \rightarrow-\infty$ and $F_{\eta} / \rho \rightarrow 0$ and $Y \rightarrow 0$ as $\eta \rightarrow \infty$. In this initial mixing-layer region the velocity in the jet is not perturbed, so that the mixing layer entrains fluid only from the stagnant side, as indicated by the boundary condition $F+\eta^{2} / 2 \rightarrow 0$ as $\eta \rightarrow-\infty$.

The analysis must be modified when a uniform velocity profile $u_{i}=1$ is considered. The appropriate similarity coordinate in that case is $\eta=(r-1) / x^{1 / 2}$ and the normalized streamfunction $F(\eta)$ must be defined to give $\rho u=F_{\eta}$ and $\rho v=x^{-1 / 2}\left(\eta F_{\eta} / 2-F / 2\right)$. The resulting equations,

$$
\begin{aligned}
{\left[\mu\left(\frac{F_{\eta}}{\rho}\right)_{\eta}\right]_{\eta}+\frac{1}{2} F\left(\frac{F_{\eta}}{\rho}\right)_{\eta} } & =0 \\
\left(\rho Y_{\eta}\right)_{\eta}+\frac{1}{2} S F Y_{\eta} & =0
\end{aligned}
$$


must be integrated with boundary conditions $F-\eta \rightarrow 0$ and $Y \rightarrow 1$ as $\eta \rightarrow-\infty$ and $F_{\eta} / \rho \rightarrow 0$ and $Y \rightarrow 0$ as $\eta \rightarrow \infty$.

The mixing-layer problem defined above was solved with a non-linear shooting method. Mixing-layer profiles of mass fraction and axial velocity $F_{\eta} / \rho$ are shown as solid curves in Fig. 2 for a $\mathrm{H}_{2}$-Air mixing layer $\left(S=1.39, \varepsilon=0.07, \mu_{a}=1.944\right)$. The computation provides in particular the initial value of the air entrainment rate $\Phi(x)=-\left(\rho r^{i} v\right)$ as $r \rightarrow \infty$, given by $\Phi=$ $-F_{\infty} x^{-1 / 2} / 2$ with $F_{\infty}=F(\eta \rightarrow \infty)=2.771$ for $u_{i}=1$ and by $\Phi=-2 A^{1 / 3} x^{-1 / 3} F_{\infty} / 3$, with $F_{\infty}=2.768$ otherwise. The figure clearly shows the effect anticipated previously related to the diffusion of the light gas. Thus, in regions where $Y \sim 1$, momentum and mass fraction diffusion are similar but, as $Y$ becomes small, the effective diffusion coefficient $\rho / S$ becomes very large, so that the decay of $Y$ as $\eta \rightarrow \infty$ is much slower than that of $u$.
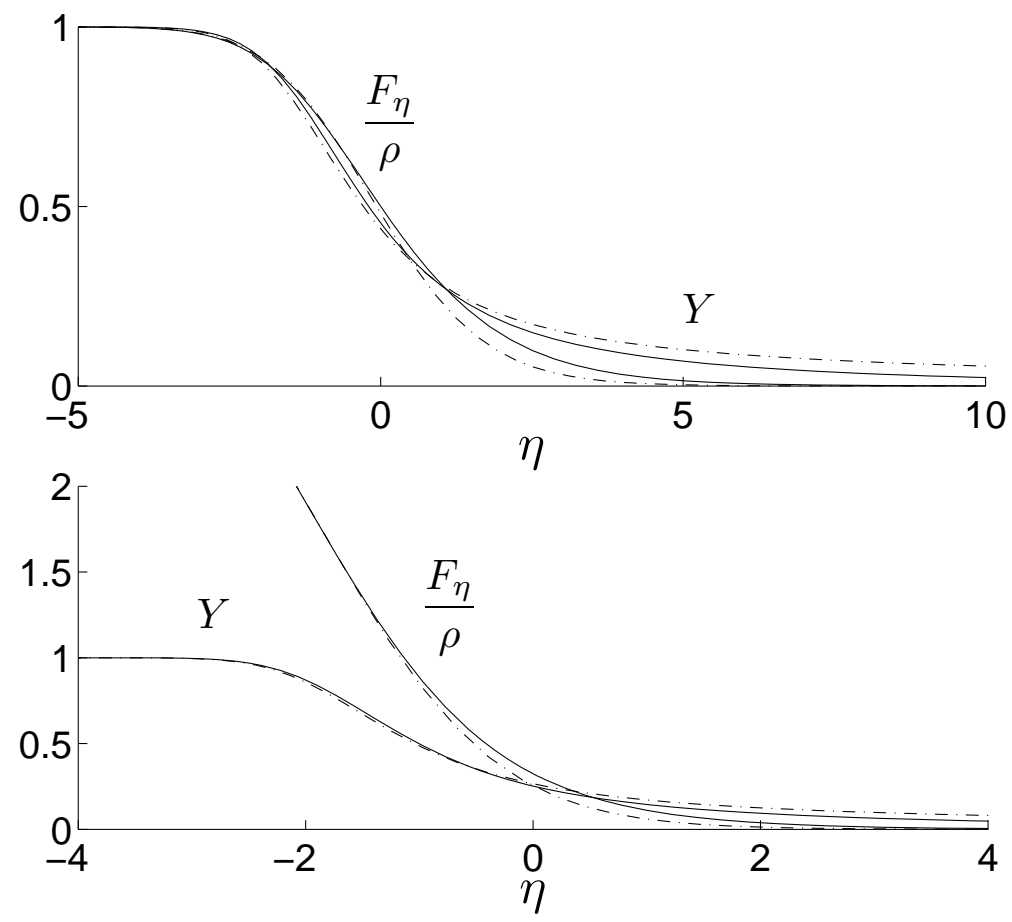

Figure 2: Velocity $u=F_{\eta} / \rho$ and mass fraction $Y$ obtained for $S=1.39$ and $\varepsilon=0.07$ (solid line) and $\varepsilon=0$ (dot-dashed line) by integration of (11) and (12) (lower plot) and by integration of (13) and (14) (upper plot).

\subsection{The boundary conditions far from the axis}

The mixing-layer solution was used in constructing the profiles of velocity and hydrogen mass fraction at $x \ll 1$, used as replacement of (6) in integrating (2)-(4). The integration employes a fully implicit marching procedure, second-order accurate in both axial and transverse directions [25], in the domain $x \in\left[\begin{array}{ll}0 & 10\end{array}\right]$ and $r \in\left[\begin{array}{ll}0 & r_{\max }\end{array}\right]$, with $r_{\max }$ taken to be sufficiently large, as explained below. A non-uniform grid has been used with a maximum clustering of points near $r=0$ and close to the jet exit $x=0$ where the minimum spacings $\delta r=4 \times 10^{-3}$ 
and $\delta x=2 \times 10^{-4}$ are utilized. The results were tested by checking that the solution satisfies everywhere the integral constraints (9) with errors smaller than $1 \times 10^{-5}$.

The treatment of the boundary conditions as $r \rightarrow \infty$ deserves specific attention. Sufficiently far from the axis, $u$ becomes negligibly small and convection is dominated by the transverse motion, with an entrainment rate $\Phi(x)=-\left(\rho v r^{i}\right)$ that is uniform in the radial direction, as can be seen from integration of (2) with $u=0$. As anticipated above, the decay of the mass fraction away from the axis is much more slow than that of $u$, so that the integration of the jet problem (2)(4) would require in principle a prohibitely large transverse integration domain $r_{\max } \gg 1$ if the condition $Y=0$ were to be imposed far from the axis. This problem can be avoided by replacing the boundary condition $Y=0$ at $r \rightarrow \infty$ with the weak condition

$$
S \Phi Y+\rho r^{i} \frac{\partial Y}{\partial r}=0
$$

evaluated at a moderately large value of $r=r_{\max }$, where the relation between the value of $Y$ and its radial gradient is obtained by integrating once (4). The numerical solution obtained by imposing $u=0$ together with (15) at $r=r_{\max }$ was tested to be independent of $r_{\max }$, provided a sufficiently large value was selected in the computation. The results shown below correspond in particular to $r_{\max }=(200,1000)$ for $i=(0,1)$ respectively.

The numerical solution at the boundary determines in particular the entrainment rate $\Phi(x)$ of the jet, which in turn determines the slow decay of the hydrogen mass fraction away from the axis, given by

$$
\frac{Y}{Y(1-\varepsilon)+\varepsilon}=\exp [-S \Phi \varepsilon(r-R)]
$$

for the planar jet and

$$
\frac{Y}{Y(1-\varepsilon)+\varepsilon}=\left(\frac{r}{R}\right)^{-S \Phi \varepsilon}
$$

for the round jet, as can be obtained by integrating a second time (15) with use made of (7). The function $R(x)$ is an apparent radius, of order unity, that can be obtained from the numerical solution by evaluating (16) and (17) with the value of $Y$ at $r=r_{\max }$.

\subsection{Results of integrations}

The results of the integrations of the jet problem for parametric values corresponding to hydrogen $\left(S=1.39, \mu_{a}=1.944\right.$ and $\left.\varepsilon=0.07\right)$ are shown in Figs. 3-6. Initial conditions include uniform and parabolic inlet velocity profiles. The results plotted include in Fig. 3 the associated boundary values of the entrainment rate $\Phi(x)$ and the function $R(x)$, obtained as part of the solution, which describe the behavior of the solution far from the axis. Profiles of axial velocity and hydrogen mass fraction for the planar and round jets are shown as solid curves in Figs. 4 and 5, whereas the evolution of their peak values, achieved along the axis, are given in Fig. 6.

As seen in Fig. 6, the streamwise decay of both $u$ and $Y$ is clearly faster for the round jet than for the plane jet due to geometrical reasons, a result also found in constant-density computations [3]. The corresponding scaling laws of the velocity and density downstream from the development region, i.e., for large values of $x$, can be anticipated by noting that, provided the hydrogen mass fraction remains sufficiently larger than $\varepsilon$, the density can be expressed in the 

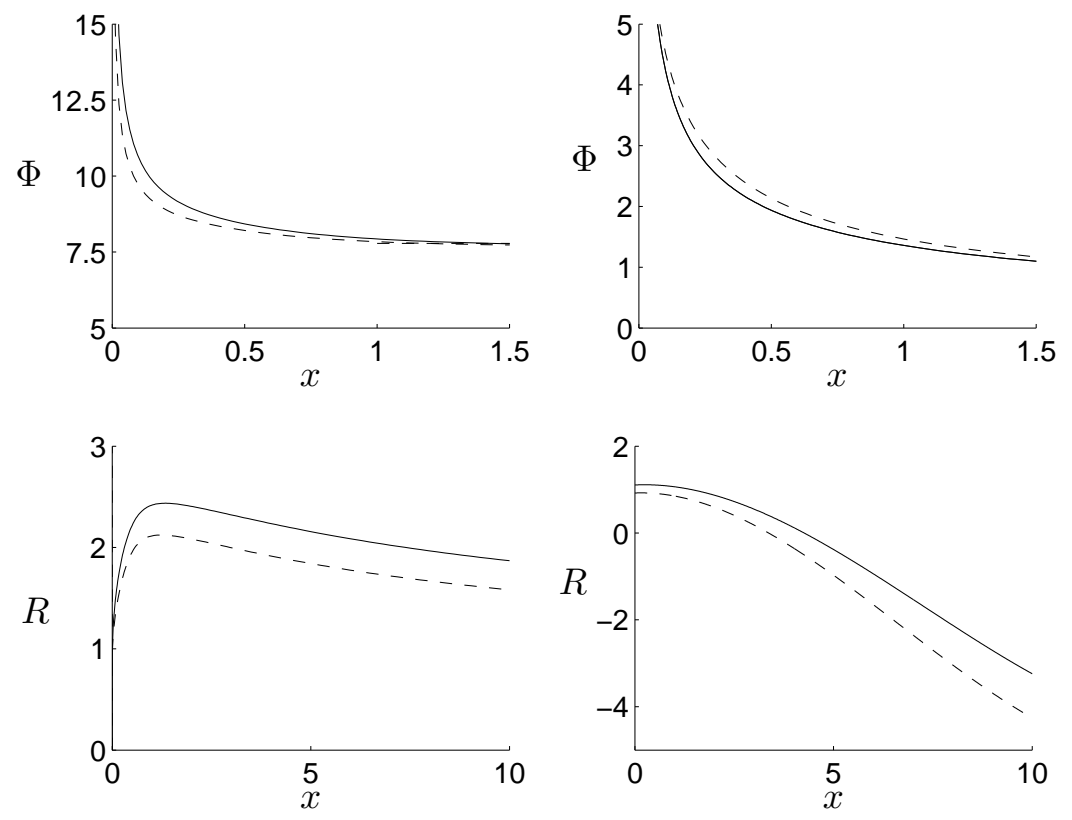

Figure 3: The functions $\Phi(x)$ and $R(x)$ as obtained from integration of (2)-(4) with $S=1.39, \mu_{a}=1.944$ and $\varepsilon=0.07$ for planar $i=0$ (right plots) and axysimmetric $i=1$ (left plots) jets with uniform (solid curves) and parabolic (dashed curves) inlet velocity profiles.

simplified form $\rho=Y^{-1}$, so that the integral constraints (9) yield the order-of-magnitude estimates $\rho u^{2} r^{1+i} \sim u r^{1+i} \sim O(1)$. When these two relationships are used together with the condition that acceleration and viscous stresses be comparable in (3), the scalings

$$
r \sim \sqrt{x}, Y \sim u \sim \rho^{-1} \sim x^{-(1+i) / 2}
$$

are obtained. Clearly, these scaling laws remain valid provided $Y \gg \varepsilon$, so that the far-field solution defined by (18) applies for $x$ in the intermediate range $1 \ll x \ll \varepsilon^{-2 /(1+i)}$. At distances $x \sim \varepsilon^{-2 /(1+i)}$ the density becomes comparable to that of the ambient and farther downstream, that is, at distances $x \gg \varepsilon^{-2 /(1+i)}$, the hydrogen mass fraction becomes much smaller than $\varepsilon$, so that, in the first approximation, the equation of state (7) reduces to $\rho=\varepsilon^{-1}$. The constant-density solutions of Schlichting and Bickley apply at these very large distances $x \gg \varepsilon^{-2 /(1+i)}$, with the scalings $r \sim \varepsilon^{1 / 3} x^{2 / 3}$ and $Y \sim u \sim \varepsilon^{1 / 3} x^{-1 / 3}$ for $i=0$ and $r \sim \varepsilon^{1 / 2} x$ and $Y \sim u \sim x^{-1}$ for $i=1$ replacing in this case those given in (18).

\section{The asymptotic limit $\varepsilon \rightarrow 0$}

It is of interest to exploit further the smallness of $\varepsilon$ by considering the limit $\varepsilon \rightarrow 0$, which will be used in particular to derive simple analytical descriptions for the far-field velocity and hydrogen mass fraction. The simplifications begin by noting that, in the region where the hydrogen mass fraction is of order unity, the equation of state (7) can be written with small errors of order $\varepsilon$ 


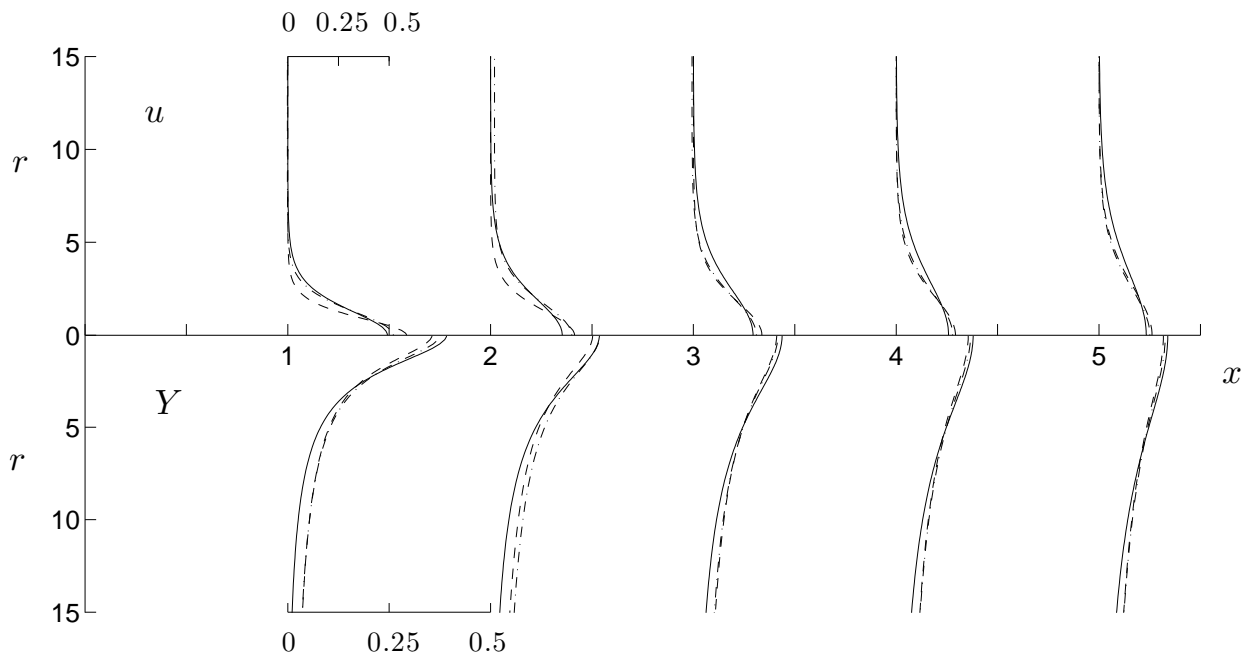

Figure 4: Numerical profiles of velocity $u$ (upper half) and mass fraction $Y$ (lower half) at different axial locations of the planar jet $(i=0)$ as obtained from integration of (2)-(4) with $S=1.39, \mu_{a}=1.944$ and initial parabolic velocity profile for $\varepsilon=0.07$ (solid curves) and $\varepsilon=0$ (dot-dashed curves). The asymptotic expressions given in (25) and (32) are represented with dashed curves.

as $\rho=Y^{-1}$. Observation of (8) also indicates that, in this same region, the viscosity is, in the first approximation, that of hydrogen, so that $\mu=1$ with small errors of order $\varepsilon$. The leading-order description in the limit $\varepsilon \rightarrow 0$ therefore involves the integration of (2)-(4) for $\rho=Y^{-1}$ and $\mu=1$ with symmetry conditions at the axis and with $u=0$ and the weak boundary conditions (15) at a large radial location. Integration is initiated at $x \ll 1$ with use made of the mixing-layer profiles determined by integrating (11)-(14) for $\varepsilon=0$, which are included in Fig. 2 for completeness.

The results obtained in the limit of vanishing jet density, independent of $\varepsilon$ and $\mu_{a}$, are included as dot-dashed curves in Figs. 4-6. As can be seen, the predictions obtained near the axis are quite satisfactory, despite the expected errors, of order $\sqrt{\varepsilon}$, associated with the assumption of constant viscosity. The accuracy degrades however away from the axis, as the value of $Y$ decreases, leading first to the failure of the approximation $\mu=1$ as $Y$ reaches values of order $\sqrt{\varepsilon}$ and then to the failure of the approximation $\rho=Y^{-1}$ as $Y \sim \varepsilon$. The expressions (16) and (17) can be used to estimate the radial extent of validity of the different approximations, yielding for instance $r \sim 1 /(S \phi \varepsilon)$ for the planar jet and $r \sim \exp [-1 /(S \phi \varepsilon)]$ for the round jet as the radial distance required for $Y$ to reach values of order $\varepsilon$, for which the approximation $\rho=Y^{-1}$ is no longer valid.

The far-field jet development at distances $x \gg 1$ can also be addressed in this limit of vanishing jet density by employing the scales identified above in (18) in defining the corresponding self-similar problem. The set of rescaled variables include the similarity coordinate $\xi=r / \sqrt{x}$, the reduced mass fraction $y=x^{(i+1) / 2} Y$ and the stream function $\psi=x^{(i+1) / 2} G$, the latter defined such that

$$
\rho r^{i} u=\frac{\partial \psi}{\partial r}=x^{i / 2} G_{\xi} \quad \rho r^{i} v=-\frac{\partial \psi}{\partial x}=\frac{1}{2} x^{(i-1) / 2}\left[\xi G_{\xi}-(1+i) G\right],
$$

where the subscript $\xi$ denotes differentiation with respect to this variable. In the self-similar 


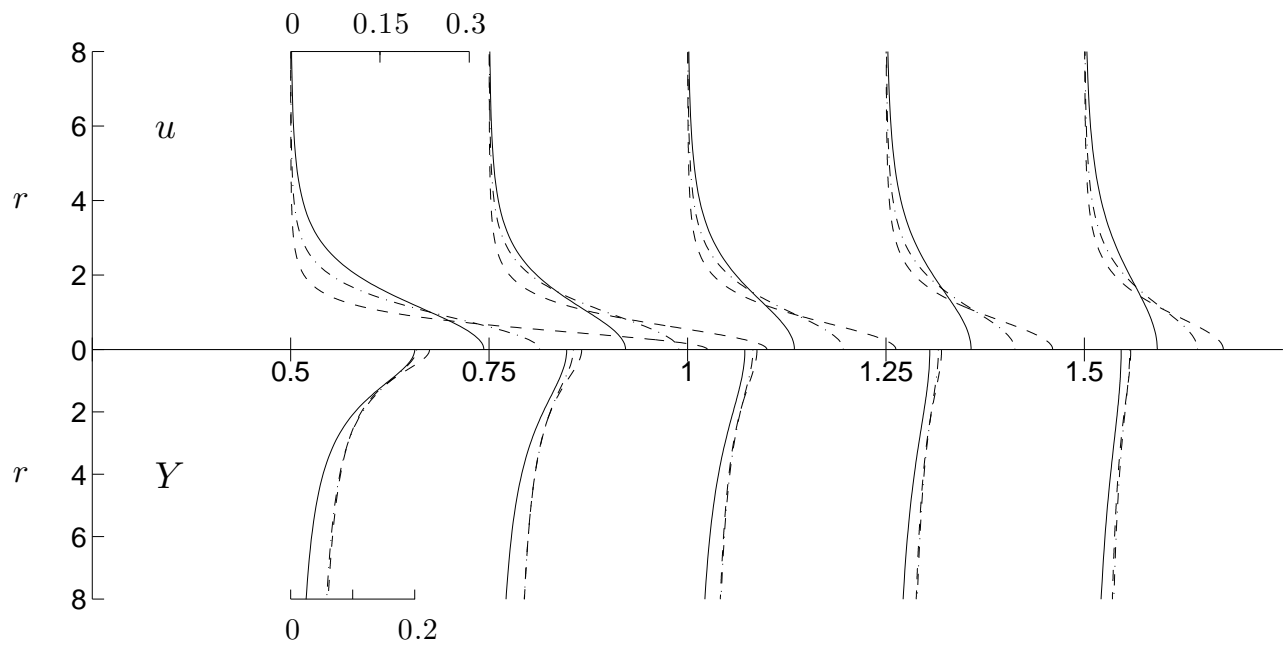

Figure 5: Numerical profiles of velocity $u$ (upper half) and mass fraction $Y$ (lower half) at different axial locations of the round jet $(i=1)$ as obtained from integration of (2)-(4) with $S=1.39, \mu_{a}=1.944$ and initial uniform velocity profile for $\varepsilon=0.07$ (solid curves) and $\varepsilon=0$ (dot-dashed curves). The asymptotic expressions given in (26) and (33) are represented with dashed curves.

formulation, the integral constraints (9) become

$$
\int_{0}^{\infty}(1+i)\left(y G_{\xi}^{2} / \xi^{i}\right) \mathrm{d} \xi=1 \text { and } \int_{0}^{\infty}(1+i) y G_{\xi} \mathrm{d} \xi=q .
$$

The problem is solved by introducing expansions for $G$ and $y$ of the form $G(\xi, x)=G_{0}(\xi)+$ $x^{-(1+i) / 2} G_{1}(\xi)+\cdots$ and $y(\xi, x)=y_{0}(\xi)+x^{-(1+i) / 2} y_{1}(\xi)+\cdots$ into (3) and (4) and solving sequentially for the different expansion terms with use made of (20) in determining the integration constants. In the development, a constant viscosity $\mu=1$ is assumed in (3), which implies that the description applies strictly only in the region $1 \ll x \ll \varepsilon^{-1 /(1+i)}$ where $Y$, much smaller than unity, is still larger than $\sqrt{\varepsilon}$.

At leading order, the problem reduces to that of integrating

$$
\begin{aligned}
\left(\xi^{i}\left(\frac{y_{0} G_{0_{\xi}}}{\xi^{i}}\right)_{\xi}\right)_{\xi}+\frac{1+i}{2}\left(G_{0} \frac{y_{0} G_{0_{\xi}}}{\xi^{i}}\right)_{\xi} & =0 \\
\frac{1}{S}\left(\xi^{i} \frac{y_{0_{\xi}}}{y_{0}}\right)_{\xi} & =0
\end{aligned}
$$

with boundary conditions $G=\left(G_{\xi} / \xi^{i}\right)_{\xi}=y_{\xi}=0$ at $\xi=0$ and $G_{\xi}=y=0$ as $\xi \rightarrow \infty$. Because of the relatively small density existing in this far-field region, transverse diffusion is the dominant transport mechanism for hydrogen. Correspondingly, the solution for (22) indicates that $y_{0}$ is constant, as corresponds to a leading-order mass fraction description $Y=x^{-(i+1) / 2} y_{0}$ 

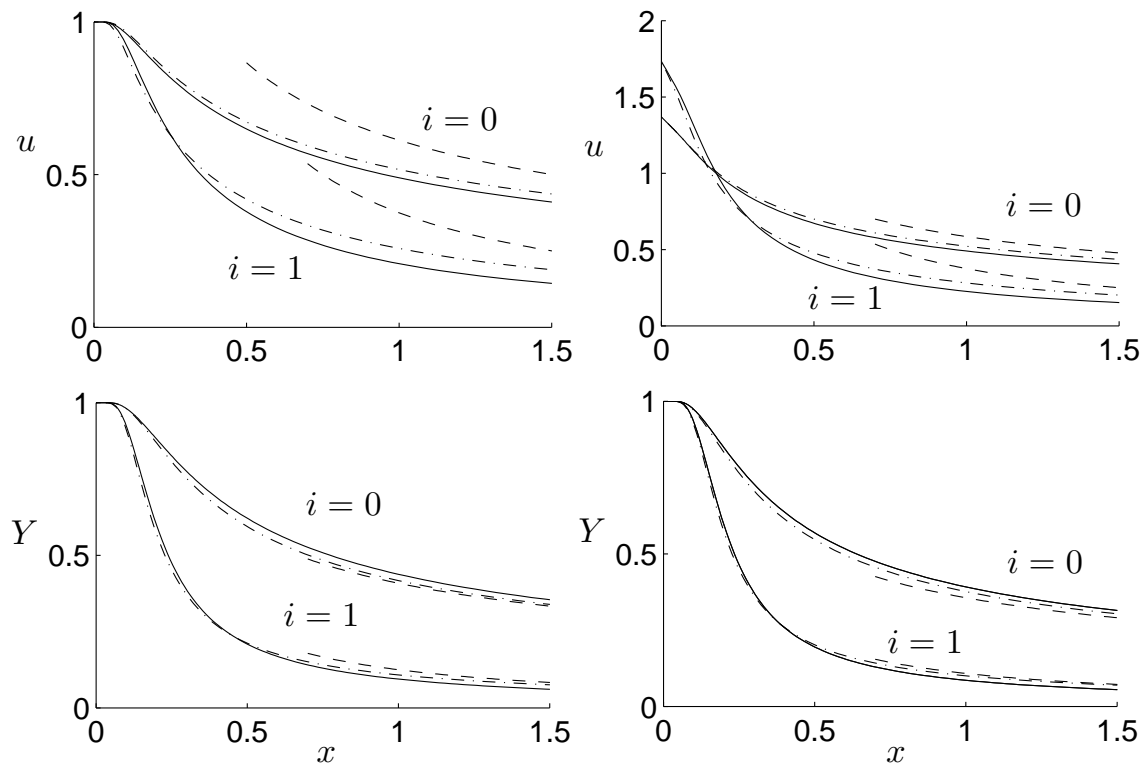

Figure 6: The evolution of the velocity $u$ and mass fraction $Y$ along the axis as obtained from integration of (2)-(4) with $\varepsilon=0.07$ (solid curves) and $\varepsilon=0$ (dot-dashed curves) for uniform (left plots) and parabolic (right plots) inlet velocity profiles. The asymptotic far-field expressions given in (27) and (28) are included in the figure with dashed curves.

that is uniform in the radial direction. Using this result in integrating (21) gives

$$
\begin{aligned}
G_{0}=(6 / q)^{1 / 2} \tanh \left[(6 / q)^{1 / 2} \xi / 4\right] & i=0 \\
G_{0}=\frac{4 \xi^{2}}{8 q / 3+\xi^{2}} & i=1,
\end{aligned}
$$

after the condition $G_{0}(\infty)=q \int_{0}^{\infty}\left(G_{0_{\xi}}^{2} / \xi^{i}\right) \mathrm{d} \xi$, obtained at this order from (20), is applied. The second integral constraint in (20) can be used to determine the constant $y_{0}=q /\left[(1+i) G_{0}(\infty)\right]$, giving $y_{0}=q^{3 / 2} / \sqrt{6}$ and $q / 8$ for $i=(0,1)$, respectively. Note that, although the functional forms obtained for the stream function $G_{0}$ in (23) and (24) may appear to be equivalent to those of Bickley and Schlichting, respectively, they are fundamentally different in that both involve a similarity variable $\xi=r / \sqrt{x}$ that does not correspond to those of the constant-density jets, where $r \sim x^{1 / 3}$ and $r \sim x$ for $i=(0,1)$, respectively.

These leading-order results can be used to construct the axial velocity profiles

$$
u=\frac{[(3 q) /(8 x)]^{1 / 2}}{\cosh ^{2}\left\{[3 /(8 q)]^{1 / 2}\left(r / x^{1 / 2}\right)\right\}} \text { for } i=0
$$

and

$$
u=\frac{8 q^{2} /(3 x)}{\left(8 q / 3+r^{2} / x\right)^{2}} \text { for } i=1,
$$

which are plotted in Figs. 4 and 5 along with the results of the numerical integrations. As can be seen, the agreement is reasonable, with discrepancies, larger for the round jet, being especially 
noticeable away from the axis, where the approximations leading to (25) and (26) can be expected to fail, as discussed above. The accompanying predictions for the downstream evolution of peak velocity and mass fractions in the planar jet

$$
u=\left(\frac{3 q}{8 x}\right)^{1 / 2} \text { and } Y=\left(\frac{q^{3}}{6 x}\right)^{1 / 2}
$$

and in the round jet

$$
u=\frac{3}{8 x} \text { and } Y=\frac{q}{8 x}
$$

are tested in Fig. 6. As can be seen, the near-axis behavior is well described by the limit of vanishing jet density, so that the expressions given in (27) and (28) constitute a sufficiently accurate representation for the jet evolution.

The analysis must be carried to the following order to enable radial variations of $Y$ to be described. The correction $y_{1}$ can be obtained by integration of

$$
\frac{1}{S}\left(\xi^{i} y_{1_{\xi}}\right)_{\xi}+\frac{1+i}{2} y_{0}^{2} G_{0_{\xi}}=0
$$

to give

$$
\begin{array}{ll}
y_{1}-y_{1}(0)=-S\left(q^{3} / 3\right) \ln \left\{\cosh \left[(6 / q)^{1 / 2} \xi / 4\right]\right\} & i=0 \\
y_{1}-y_{1}(0)=-S\left(q^{2} / 32\right) \ln \left[1+3 \xi^{2} /(8 q)\right] & i=1,
\end{array}
$$

where the correction at the axis $y_{1}(0)$ would be determined in terms of the value of $G_{1}(\infty)$ form the integral constraint $y_{0} G_{1}(\infty)+\int_{0}^{\infty} G_{0_{\xi}} y_{1} \mathrm{~d} \xi=0$, obtained at this order from the second equation in (20).

The two-term expansion $y=y_{0}+x^{-(1+i) / 2} y_{1}$ improves predictions of hydrogen mass fraction near the axis. The diverging character of $y_{1}$ for increasing values of $\xi$ limits however its applicability to the region where $\xi$ is of order unity, corresponding to radial distances $r \sim \sqrt{x}$. In constructing a uniformly valid expression for $Y$, applicable also at large distances from the axis, it is therefore better to use the alternative expression $y=y_{0} /\left(1-x^{-(1+i) / 2} y_{1} / y_{0}\right)$, which is equivalent to the two-term expansion $y=y_{0}+x^{-(1+i) / 2} y_{1}$ for $\xi \sim O(1)$ but ensures a decaying $\mathrm{H}_{2}$ mass fraction as $\xi \rightarrow \infty$. If the small correction $y_{1}(0)$ is further neglected for simplicity, the corresponding expressions for $Y$ simplify to

$$
\begin{array}{ll}
Y=\frac{q^{3 / 2} /(6 x)^{1 / 2}}{1+\left[2 S q^{3 / 2} /(6 x)^{1 / 2}\right] \ln \left\{\cosh \left[(3 /(8 q))^{1 / 2}\left(r / x^{1 / 2}\right)\right]\right\}} & i=0 \\
Y=\frac{q /(8 x)}{1+[S q /(4 x)] \ln \left[1+3 r^{2} /(8 q x)\right]} & i=1 .
\end{array}
$$

The accuracy with which these expressions describe the hydrogen mass fraction is tested in Figs. 4 and 5. As can be seen, the agreement is reasonably good, with the results of the planar case being in particular very accurate over the whole range of $x$ considered.

\section{Conclusions}

Numerical and asymptotic methods have been employed to investigate the structure of planar and round hydrogen jets with full account of the variation with composition of the density and 
transport properties. The information provided serves to characterize in detail the velocity and mass fraction fields in laminar configurations with moderately large jet Reynolds numbers, $R_{j}$, for which the jet remains slender and stable. Profiles of axial velocity $u$ and hydrogen mass fraction $Y$ in the near field and also in the jet development region are computed in the boundarylayer approximation, given the results shown in Figs. 2, 4 and 5. The downstream evolution of the accompanying peak values of $u$ and $Y$, found along the axis, is plotted in Fig. 6. Analytical expressions are given in (16) and (17) for the radial decay of $Y$ away from the axis, where the functions $R(x)$ and $\Phi(x)$ are plotted in Fig. 3

The analysis has exploited the small value $\varepsilon=W_{j} / W_{\text {air }}=0.07$ of the hydrogen-to-air molecular weight ratio, by considering the asymptotic limit of vanishing jet densities. This limit was demonstrated to give an approximate description, independent of $\varepsilon$ and of the air viscosity, that describes with sufficient accuracy many aspects of the hydrogen jet. In particular, the development provides simple explicit expressions for the velocity and mass fraction, which are summarized in (25)-(28), (32) and (33). These expressions are valid in the intermediate far-field region where $Y$ is much smaller than unity and still much larger than $\varepsilon^{1 / 2}$, corresponding to distances from the jet exit $x^{\prime}$ in the range $1 \ll x^{\prime} /\left(R_{j} a\right) \ll \varepsilon^{-1 /(1+i)}$, where $a$ is the initial jet radius.

\section{Acknowledgements}

This work was supported by the Spanish MICINN under Project \# ENE2008-06515-C04 and by the Comunidad de Madrid under Project S2009/ENE-1597 (HYSYCOMB)

\section{References}

[1] M.F. El-Amin, M. Inoue, H. Kanayama, Boundary layer theory approach to the concentration layer adjacent to a ceiling wall of a hydrogen leakage: Far region, International Journal of Hydrogen Energy, 33, (2008), 7642-7647

[2] El-Amin MF, Kanayama H, Similarity consideration of the buoyant jet resulting from hydrogen leakage, Int J Hydrogen Energy 34 (2009), pp. 5803-5809

[3] Revuelta, A., Sánchez, A. L., Liñán, A., The Virtual Origin as a First-Order Correction for the Far-Field Description of Laminar Jets, Phys. Fluids, (2002) 14, 1821-1824.

[4] V. K.Garg, Spatial stability of the non-parallel Bickley jet, J. Fluid Mech., 102, 127-140 (1981).

[5] P. J. Morris, The spatial viscous instability of axisymmetric jets, J . Fluid Mech., 77, 511-529 (1976)

[6] Andrade, E. N. \& Tsien, L. C. The velocity distribution in a liquid into liquid jet. Proc. Phil. Soc. London 49, 381-391 (1937)

[7] P. O'Neill, J. Soria, D. Honnery, The stability of low Reynolds number round jets, Experiments in Fluids 36 (2004) 473483

[8] G. W. Rankin, K. Sridhar, M. Arulraja, K. R. Kumar, An experimental investigation of laminar axisymmetric submerged jets, J. Fluid Mech., 133, 217-231 (1983).

[9] A. J. Reynolds, Observations of a liquid-into-liquid jet, J. fluid Mech., 14, 552-556 (1962)

[10] D. Koller-Milojevic and W. Schneider, Free and confined jets at low Reynolds numbers, Fluid Dynamics Research, 12, 307- -322 (1993)

[11] Andrade, E. N. The velocity distribution in a liquid into liquid jet. The plane jet. Proc. Phil. Soc. London 51, 784-793 (1939)

[12] Schlichting, H., Laminare strahlenausbreitung, Z. Angev. Math. Mech. (1933) 13, 260-263.

[13] Bickley, W., The plane jet, Phil. Mag. (1937) 23, 727-731.

[14] Sánchez-Sanz M., Sánchez A. L., Liñán A., Fronts in high-temperature laminar gas jets, J. Fluid Mech. (2006) , $547,257-266$

[15] Sánchez-Sanz M., Sánchez A. L., Liñán A., Variable-Density Jet Flows Induced by Concentrated Sources of Momentum and Energy, Theoretical and Computational Fluid Dynamics, submitted (2009)

[16] Pai, S. I., Fluid Dynamics of Jets, (1954) D. Van Nostrand Company, Toronto.

[17] Chou, P.Y., The laminar mixing motion of two incompressible gases, Chinese J. Phys., (1947) 2, 96-101

[18] Crane, L. J. \& Pack, D. C., The Mixing of a Jet of Gas with an Atmosphere of a Different Gas at Large Distances from the Orifice.Part I: The Plane Jet., Quat. Journ. Mech. and Applied Math. 1961 14(4):385-391. 
[19] Crane, L. J. \& Pack, D. C., The Mixing of a Jet of Gas with an Atmosphere of a Different Gas at Large Distances from the Orifice.Part I: The round Jet., Quat. Journ. Mech. and Applied Math. 1961 14(4):393-402 .

[20] Maydew, R.C., Reed, J.F., Turbulent mixing of compressible free jets, AIAA Journal, (1963), 1, 6, 1443-1444

[21] Crane, L. J., Axially Symmetric Plumes at Very Small Pradtl Numbers, J. Appl. Math. Phys. (ZAMP) (1975) 26, 427-435

[22] Hirschfelder J.O., Curtiss C.F., Bird R.B. Molecular Theory of Gases and Liquids. New York, J. Wiley 1954

[23] Rosner D.E. Trasport Processes in Chemically Reacting Flows, Dover, 2000

[24] Wilke C.R. A Viscosity Equation for Gas Mixtures, J. Chem. Phys. (1950), 18, 517-519

[25] Anderson D. A., Tannehill J. C., Pletcher R.H.. Computational Fluid Mechanics and Heat Transfer, Hemisphere publishing corporation, New Yor, 1984. 